\title{
Introductory notes for the Acta IMEKO Special Section on the "21st Symposium on Measurement of Electrical Quantities" and the "19th Workshop on ADC/DAC Modelling and Testing"
}

\author{
Alexandru Salceanu, Vilmos Pálfi \\ "Gheorghe Asachi" Technical University of Iasi, Mangeron Str. 67, 700050, Iasi, Romania \\ Budapest University of Technology and Economics, Magyar tudósok krt. 2. I, 1117, Budapest, Hungary
}

\begin{abstract}
Section: Editorial
Citation: Alexandru Salceanu, Vilmos Pálfi, Introductory notes for the Acta IMEKO Special Section on the "21st Symposium on Measurement of Electrical Quantities" and the "19th Workshop on ADC/DAC Modelling and Testing", Acta IMEKO, vol. 6, no. 4, article 1, December 2017, identifier: IMEKO-ACTA-06 (2017)-04-01
\end{abstract}

Section Editor: Paul Regtien, Measurement Science Consultancy, The Netherlands

Received: November 24, 2017; In final form: November 24, 2017; Published December 2017

Copyright: (C) 2017 IMEKO. This is an open-access article distributed under the terms of the Creative Commons Attribution 3.0 License, which permits unrestricted use, distribution, and reproduction in any medium, provided the original author and source are credited

Corresponding author: AlexandruSalceanu, e-mail: asalcean@tuiasi.ro

\section{INTRODUCTION}

Number 4, Volume 6 of Acta IMEKO is submitted to your assessments and suggestions. This issue is centered on the $21 \mathrm{st}$ Symposium on Measurement of Electrical Quantities and 19th Workshop on ADC/DAC Modelling and Testing which were held in the city of Budapest, Hungary on September 2016. The main objectives of these scientific tribute and events were to present the latest research, to stimulate information exchanges on current research dedicated to the measurement, monitoring and recording of electrical signals.

Since October till December 2016, the members of the IMEKO TC4 Board identified 11 valuable contributions presented in the Budapest events, for eventual publication in Acta IMEKO. Their authors were invited in December 2016 to submit extended and updated versions of their selected papers. All of the authors that gave positive responses to this challenge started sending their extended papers to the Acta IMEKO online submission system early 2017. A significant number of representative reviewers proceeded in successive stages, their task of assessing the papers and submitting their recommendations. The final list of 8 published papers in this issue is a high-quality exhibition of the first-class papers submitted to our Symposium. All the reviewers involved in the process gave their support aiming to further improve not only the shape but also the intrinsic content of the papers.

We sincerely hope that all the contributions you will find in the next pages provide you up-to-date information of your interest, being an invitation to continue and develop your multilevel partnership with Acta IMEKO.

\section{ABOUT TC4}

The key, vital interest and activity of IMEKO TC4 (measurement of electrical quantities) is the development of theoretical and practical research in the field of electrical and electronic measurements. This Technical Committee organizes each year, since its inception in 1984, specific symposia and workshops. The continued boost in general relevance and the inclusion of electrical and electronic tools for the measurement, monitoring and recording of electrical signals in our daily lives has significantly improved our activities and increased the world-wide addressability.

The current members of TC4 are:

Dominique Dallet, Chairperson - France

Pedro M. Ramos, Deputy Chairperson -Portugal

Alexandru Salceanu, Scientific Secretary - Romania

Linus Michaeli, Past Chairperson - Slovak Republic

Pasquale Daponte, Past Chairperson - Italy

Antonio Manuel da Cruz Serra, Past Chairperson -Portugal

Janusz Mindykowski, Past Chairperson - Poland

Mario Savino, Honorary Chairperson - Italy

Raul Land - Estonia

Joaquin Del Rio Fernández - Spain

Turgay Özkan - Turkey

Umberto Pogliano - Italy

Gelson Rocha - Brazil

Jan Saliga - Slovak Republic

Dragana Popovic Renella-Switzerland

Sergey G. Semenchinsky - Russia

Yurij Tesyk - Ukraine 
Olfa Kanoun - Germany

Dušan Agrež - Slovenia

Victor I. Didenko - Russia

Izzet Kale - United Kingdom

Leo van Biesen - Belgium

Laurent Francis-Belgium

Mihai Cretu - Romania

Christian Eugène - Belgium

Sohair Fakhry - Egypt

Vladimir Haasz - Czech Republic

Jouko Halttunen - Finland

Peter Händel - Sweden

Damir Ilic - Croatia

Voicu Groza - Canada

Elefterios Kayafas - Greece

Wang Xiaofei - China

He Qing - China

Platon Sovilj-Serbia

Hyung Kew Lee-Republic of Korea

\section{THE ARTICLES}

The paper submitted by Zsolt Kemény et al (p.5) presents developed research focused on the dependence of work-surface resistance on ambient conditions and surface contamination in a live industrial environment, relying on an automated facility management. It is beneficial that the results have been taken without interfering with the working system, while using contactless and remote techniques. The model of surface resistance is based on data acquired during one year of various measurements.

The paper presented by Marco Crescentini et al (p.17) is mainly an experimental research based on a solid theoretical foundation on the evaluation of the upper limit of Hall sensors' bandwidth. In the paper an innovative technique is developed, specially dedicated to estimating the response time of the Hall sensor, proposing an equivalent electric model for its dynamic response. A very useful differentiation between the upper limit of the bandwidth determined by intrinsic capacitance and the more practical one imposed by the general input capacitance of the reading interface is sustained.

The hardware and software implementation in LabVIEW of a low-cost multichannel interface designed for passive resistive sensors is detailed in the paper put forward by Zivko Kokolanski et al (p.25). The use of a monostable configuration for the multivibrator oscillator has the advantage of direct interfacing with FPGAs or microcontrollers. The presented details of sensor interfacing and calibration techniques are convincing, being also supported by the practical realization of a prototype multichannel resistive sensor interface that could be a very suitable solution when a medium accuracy, low-cost and small-sized implementation is desirable.

A compact size, high-precision amplifier for a strain-gauge transducer is here provided by André Schäfer (p.31). The argumentation for the use of inductive dividers (based on the ratio of the number of windings of the coil) versus resistive dividers to the realization of high precision amplifiers involved for primary calibration purposes is detailed. A compact dualchannel module is presented which can process and display multiple signals, with different requirements for accuracy and bandwidths. The very good time and temperature stability of the amplifiers is proved by the performed measurements.

The general approach proposed by Alexandru Salceanu et al (p.37) is developed around an affordable determination of low frequency electric fields, from the perspective of SM enterprises, which have to comply with the restrictions imposed by Directive 2013/35/EU. The mechanisms by which electric fields interact with humans are reviewed, their values and limitations being comparatively analyzed, while respecting the documentation elaborated by different specialized bodies. Constructive details of two laboratory-made suitable E-field sensors are also described. The case study detailing a cumulative assessment methodology for evaluating the exposure to electric fields with different frequencies is complemented by modeling the electric field in the vicinity of an overhead High Voltage Line with specific configuration, based on free, open access software.

The topic of improving the automated lung screening for acquiring depth information, without high exposure to X-rays, at affordable cost is dealt by Bence Tilk (p.46).A very good compromise between time, cost and exposure to ionizing radiations could be the Digital Tomosynthesis (DTS). The paper presents solutions for solving the main problem of DTS, the blurred slice image that makes it hard to automatically segment the lung areas, essential requirement for an efficient Computer-aided Diagnosis system. A combined approach is proposed by using snake methods and adjacent image segmentation information to improve lung screening and to provide robust solutions. Three different model-free approaches are used to take advantage of each method on different areas of the lungs: intensity-based segmentation for center point identification, gradient-based segmentation for non-central points of the lung and snake for segment the outer side of the lung. The advantage of using more complex models is to gain information from adjacent images to improve the incorrectly segmented region on the non-central images.

An improvement of the estimation of the signal components from random equivalent and non-coherent sampling measurements is provided by Dušan Agrež (p.54). A balance between theory and experiment is submitted, by demonstrating the behavior of estimations of the periodic signal parameters in the case of random sampling and under non-coherent sampling condition in a real measurement environment. Aiming to achieve the lowest estimation errors, the non-parametric IDFT estimation of frequency is here used first for detection of the component frequency in both the parametric and nonparametric approach and complementary, the iterative parametric method has been used for other parameters when the sampling frequency is relatively lower than the Nyquist frequency and the sampling procedure becomes pure random.

Simulations and experimental results demonstrated that the estimations of the components frequency, amplitude and phase are possible when the duty ratio of the random samples from the total number of samples in the non-coherent measurement interval is very low.

In paper (p.61) László Sujbert et al introduced an identification procedure for random independent, random block-based and general Markov model-based data loss patterns. In this paper the authors proposed a quasi-inverse procedure, by comparison with the mostly encountered one: the data loss model can be identified by the Fourier transform of the data availability indicator function. First the Power Spectral Density of the indicator function was calculated and then a parametric system identification method has been used to get the spectral parameters. By comparing the measurement spectra to the model spectra, the model order selection and 
verification can be also easily done. The developed method provides not only an estimation of the loss signal, but also makes an estimation of the parameters of the data loss model available.

\section{CONCLUSIONS}

It was a great honor for us to act as Guest Editors for an issue of Acta IMEKO, both from the perspective of working for a high-profile scientific journal and also, of promoting TC4's great tradition symposia, now reaching the 21 st edition. One of us had previously the privilege of working as Guest Editor for a special issue of Acta IMEKO in 2013, after the
Barcelona TC4 symposium. We have noted with satisfaction the increase in quality in general, mainly the higher flexibility of the electronic submission platform, the constructive exigencies of the reviewers' team, elevated standards for copyediting, proofreading and layout editing services. But, first of all, we had to take notice of the superior quality of end-accepted articles for publication.

A special appreciation for the two Editors in Chief, Paul P.L. Regtien (who, even if "Honorary", continues to be very active, prompt and involved) and Paolo Carbone, whom I felt completely devoted to this wonderful ascendant project called Acta IMEKO. 